\title{
How neutral is the intergalactic medium surrounding the redshift $z=7.085$ quasar ULAS J1120+0641?
}

\author{
J. S. Bolton, ${ }^{\star \star}$ M. G. Haehnelt, ${ }^{2}$ S. J. Warren, ${ }^{3}$ P. C. Hewett, ${ }^{2}$ D. J. Mortlock, ${ }^{3}$ \\ B. P. Venemans, ${ }^{4}$ R. G. McMahon ${ }^{2}$ and C. Simpson ${ }^{5}$ \\ ${ }^{1}$ School of Physics, University of Melbourne, Parkville, VIC 3010, Australia \\ ${ }^{2}$ Kavli Institute for Cosmology and Institute of Astronomy, Madingley Road, Cambridge CB3 OHA \\ ${ }^{3}$ Astrophysics Group, Imperial College London, Blackett Laboratory, Prince Consort Road, London SW7 2AZ \\ ${ }^{4}$ European Southern Observatory, Karl-Schwarzschild Strasse 2, 85748 Garching bei München, Germany \\ ${ }^{5}$ Astrophysics Research Institute, Liverpool John Moores University, Twelve Quays House, Egerton Wharf, Birkenhead CH41 1LD
}

Accepted 2011 June 23. Received 2011 June 22; in original form 2011 May 2

\begin{abstract}
The quasar ULAS J1120+0641 at redshift $z=7.085$ has a highly ionized near zone which is smaller than those around quasars of similar luminosity at $z \simeq 6$. The spectrum also exhibits evidence for a damping wing extending redward of the systemic Ly $\alpha$ redshift. We use radiative transfer simulations in a cosmological context to investigate the implications for the ionization state of the inhomogeneous intergalactic medium (IGM) surrounding this quasar. Our simulations show that the transmission profile is consistent with an IGM in the vicinity of the quasar with a volume averaged $\mathrm{H}_{\mathrm{I}}$ fraction of $\left\langle f_{\mathrm{H}_{\mathrm{I}}}\right\rangle_{\mathrm{V}} \gtrsim 0.1$ and that ULAS J1120+0641 has been bright for $10^{6}-10^{7} \mathrm{yr}$. The observed spectrum is also consistent with smaller IGM neutral fractions, $\left\langle f_{\mathrm{H}_{1}}\right\rangle_{\mathrm{V}} \sim 10^{-3}$ to $10^{-4}$, if a damped Ly $\alpha$ system in an otherwise highly ionized IGM lies within 5 proper Mpc of the quasar. This is, however, predicted to occur in only $\sim 5$ per cent of our simulated sightlines for a bright phase of $10^{6}-10^{7} \mathrm{yr}$. Unless ULAS $\mathrm{J} 1120+0641$ grows during a previous optically obscured phase, the low age inferred for the quasar adds to the theoretical challenge of forming a $2 \times 10^{9} \mathrm{M}_{\odot}$ black hole at this high redshift.
\end{abstract}

Key words: dark ages, reionization, first stars - intergalactic medium - quasars: absorption lines.

\section{INTRODUCTION}

The spectra of quasars with redshifts $z \gtrsim 6$ are an important probe of the ionization state of the intergalactic medium (IGM) in the early Universe. The intergalactic Ly $\alpha$ opacity rapidly rises with increasing redshift, and consequently there has been much debate whether these quasars have been observed before the Universe was fully reionized (e.g. Becker, Rauch \& Sargent 2007; Lidz et al. 2007; Mesinger 2010). Studies of the small windows of Ly $\alpha$ transmission observed in close proximity to these objects provide important clues in this respect (Mesinger \& Haiman 2004; Wyithe \& Loeb 2004; Fan et al. 2006; Alvarez \& Abel 2007; Bolton \& Haehnelt 2007a; Maselli, Ferrara \& Gallerani 2009). These highly ionized 'near zones' lie between the red edge of the Gunn \& Peterson (1965) trough and the quasar Ly $\alpha$ emission line, and arise because the hydrogen close to these sources is highly ionized.

^E-mail: jsbolton@unimelb.edu.au
Mortlock et al. (2011) (hereafter M11) have recently reported the highest redshift quasar found to date, ULAS J1120+0641 at $z=7.085$, discovered using data from the UKIRT Infrared Deep Sky Survey (UKIDSS, Lawrence et al. 2007). In their analysis of the Ly $\alpha$ near zone observed in a low-resolution $(R \simeq 1400)$ VLT/FORS2 spectrum of moderate signal-to-noise ratio, M11 find the extent and shape of the near zone is distinct from those around the much debated $z \sim 6$ quasars. Assuming the quasar is embedded in a homogeneous intergalactic medium (IGM), M11 concluded the observed transmission profile of ULAS J1120+0641 is consistent with a surrounding IGM for which the volume averaged neutral fraction is $\left\langle f_{\mathrm{H}_{\mathrm{I}}}\right\rangle_{\mathrm{V}}>0.1$ at $z \simeq 7.1$.

In this Letter we examine the implications of the M11 observation in more detail using simulations of radiative transfer through an inhomogeneous IGM. These simulations have successfully explained the properties of $\operatorname{Ly} \alpha$ near zones observed at $z \simeq 6$ in the context of a highly ionized IGM (Bolton et al. 2010). We begin in Section 2 by giving a brief overview of Ly $\alpha$ near zone sizes. In Section 3 we describe our numerical simulations before comparing them directly to the M11 measurement in Section 4. Finally in 


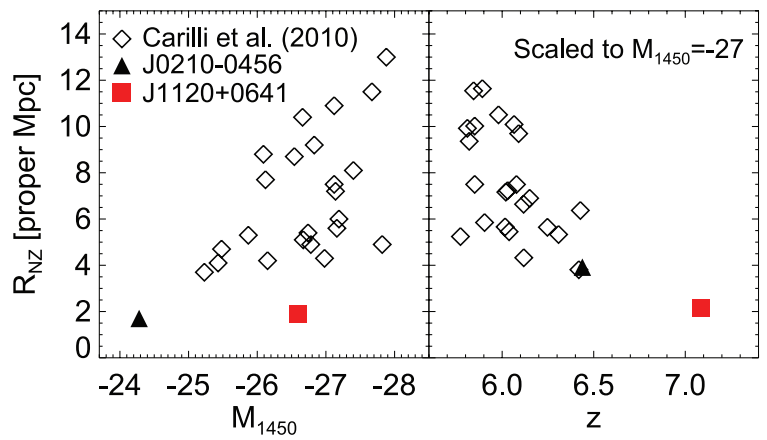

Figure 1. Summary of near zone size measurements in the current literature. Left: near zone sizes against the quasar absolute magnitude. Right: near zone sizes against redshift. The sizes have been rescaled by a factor $10^{0.4\left(27+M_{1450}\right) / 3}$ (Fan et al. 2006) to correspond to a common absolute magnitude of $M_{1450}=-27$. Note this scaling assumes $R_{\mathrm{NZ}} \propto \dot{N}^{1 / 3}$, where $\dot{N}$ is the emission rate of ionizing photons from the quasar, appropriate if the near zone traces the boundary of the quasar $\mathrm{H}$ II region. If the near zone extent is instead set by resonant absorption, $R_{\mathrm{NZ}} \propto \dot{N}^{1 / 2}$ (Bolton \& Haehnelt 2007a).

Section 5 we discuss the implications of our results and conclude. We assume the cosmological parameters $\Omega_{\mathrm{m}}=0.26, \Omega_{\Lambda}=$ $0.74, \Omega_{\mathrm{b}} h^{2}=0.023$ and $h=0.72$ throughout, and unless otherwise stated all distances are given in proper units.

\section{QUASAR NEAR ZONE SIZES}

At the luminosities typical of observed $z \simeq 6$ quasars, and assuming an optically bright phase of $t_{\mathrm{q}} \sim 10^{7} \mathrm{yr}$, the size of a quasar $\mathrm{H}$ II region expanding into a partially neutral IGM and that of a proximity zone embedded in an already highly ionized IGM are comparable (Bolton $\&$ Haehnelt 2007a). Further, if the surrounding IGM is significantly neutral, the red Gunn-Peterson (GP) damping wing will reduce the observed size of the near zone and Ly $\alpha$ absorption may extend redward of the quasar systemic redshift (Miralda-Escude \& Rees 1998; Mesinger \& Haiman 2004). Unfortunately, the possibility of confusing this damping wing with a collapsed region with a high $\mathrm{H}$ I column density in an otherwise highly ionized IGM complicates matters further. These issues make the interpretation of the Ly $\alpha$ near zones observed around these quasars with respect to the IGM ionization state ambiguous (Bolton \& Haehnelt 2007a; Lidz et al. 2007; Maselli et al. 2007).

A summary of the existing measurements of Ly $\alpha$ near zone sizes around high-redshift quasars, compiled recently by Carilli et al. (2010), is displayed in Fig. 1 . Interestingly, ULAS J1120+0641 has a small ${ }^{1}$ near zone size given its bright absolute AB magnitude, $M_{1450}=-26.6$. This is demonstrated by the evolution of observed near zone sizes with redshift, rescaled to correspond to a common magnitude of $M_{1450}=-27$, in the right-hand panel of Fig. 1. M11 have noted that the small near zone size around ULAS J1120+0641, coupled with Ly $\alpha$ absorption redward of the systemic redshift from a putative red damping wing, may indicate the IGM surrounding ULAS J1120+0641 is significantly more neutral than around quasars at $z \sim 6$. We now investigate this in more detail using realistic synthetic absorption spectra which employ a variety of evolutionary histories for the IGM ionization state.

\footnotetext{
${ }^{1}$ The only quasar with a similarly small near zone is CFHQS J0210-0456 at $z=6.44$ (Willott et al. 2010), but this object is considerably fainter and is consistent with the other $z \simeq 6$ quasars on accounting for its fainter luminosity.
}

\section{NUMERICAL SIMULATIONS}

We construct Ly $\alpha$ absorption spectra using a high-resolution hydrodynamical simulation combined with a line-of-sight radiative transfer code (Bolton et al. 2010). The hydrodynamical simulation was performed using the GADGET-3 code (Springel 2005) and has a box size of $10 \mathrm{~h}^{-1}$ comoving Mpc and a gas particle mass of $9.2 \times 10^{4} h^{-1} \mathrm{M}_{\odot}$. A total of 100 sightlines of length $96.6 h^{-1}$ comoving Mpc were extracted around haloes identified in the simulation to construct skewers through the IGM density, temperature and peculiar velocity field. The largest halo mass in the simulation volume at $z=7.1$ is $4.7 \times 10^{10} h^{-1} \mathrm{M}_{\odot}$, which likely underestimates the ULAS J1120+0641 host halo mass. However, resolving the low-density IGM is more important for modelling the Ly $\alpha$ absorption, and a small, high-resolution simulation provides the best compromise (Bolton et al. 2010). Following M11 and Bolton et al. (2010), we assume the spectrum of the unobscured, radio quiet quasar ULAS J1120+0641 is a broken power law where $f_{v} \propto v^{-0.5}$ for $1050<\lambda<1450 \AA$ and $f_{v} \propto v^{-1.5}$ for $\lambda<1050 \AA$, yielding an ionizing photon emission rate of $\dot{N}=1.3 \times 10^{57} \mathrm{~s}^{-1}$.

The ionization state of the IGM along the sightlines prior to the quasar turning on was initialized using four different models for the background $\mathrm{H}$ i photoionization rate, displayed in Fig. 2 . However, it is important to note that reionization is an inhomogeneous process, and as a result each model may be realized at the same time in different regions of the IGM (Mesinger \& Furlanetto 2008). Furthermore, the ionization state of the IGM in the vicinity of a massive host halo will be biased due the clustering of lower luminosity

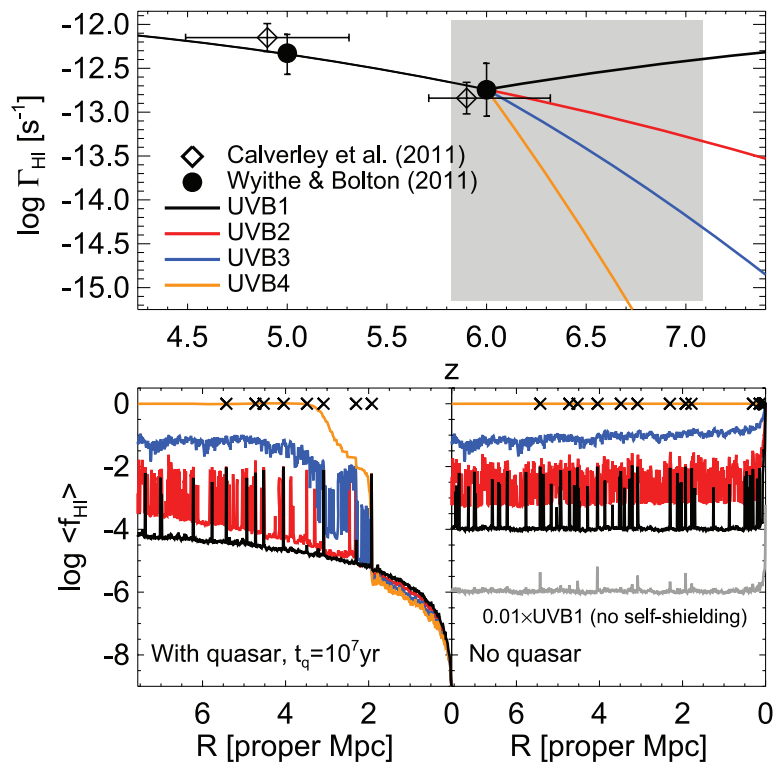

Figure 2. Top: four simple models for the redshift evolution of the background $\mathrm{H}_{\text {I }}$ photoionization rate along a quasar sightline. The grey shading represents the range covered by the $\operatorname{Ly} \alpha$ forest to rest frame $\operatorname{Ly} \beta$ for a quasar at $z=7.085$. The measurements were obtained by Wyithe \& Bolton (2011) from the Ly $\alpha$ forest opacity and by Calverley et al. (2011) using the proximity effect. Note that for UVB4 we set $\Gamma_{\mathrm{H}_{\mathrm{I}}}=0$ at $z>6$.9. Bottom: the neutral hydrogen fraction averaged over 100 simulated sightlines produced by the UVB models including (left) and excluding the quasar (right). The averaging is performed for display purposes only, and results in neutral regions being displayed as spikes with $\left\langle f_{\mathrm{H}_{\mathrm{I}}}\right\rangle \sim 10^{-2}$. The crosses mark the position of all DLAs in the sightlines constructed using UVB4, and the grey curve in the right-hand panel shows the logarithm of $\left\langle f_{\mathrm{H}_{\mathrm{I}}}\right\rangle$ for UVB1 excluding the self-shielding approximation, multiplied by a factor of 0.01 for clarity. 
Table 1. Average $\mathrm{H}_{\mathrm{I}}$ fractions and the incidence of optically thick absorbers within $0.05<R<5 \mathrm{Mpc}$ of the quasar host halo in all 100 simulated sightlines including the self-shielding prescription. The absorber columns are estimated by integrating the $\mathrm{H}_{\mathrm{I}}$ densities over a scale of $20 \mathrm{kpc}$. From left to right, the columns list the volume and mass averaged $\mathrm{H}_{\mathrm{I}}$ fraction, the number of LLSs $\left(10^{17.2}<N_{\mathrm{H}_{\mathrm{I}}}<10^{20.3} \mathrm{~cm}^{-2}\right)$ and DLAs $\left(N_{\mathrm{H}_{\mathrm{I}}}>10^{20.3} \mathrm{~cm}^{-2}\right)$ per unit redshift and the fraction of sightlines where there is at least one LLS or DLA, all prior to the quasar turning on. In the final four columns the latter are also given after a quasar with age $10^{6}$ and $10^{7} \mathrm{yr}$ has ionized the surrounding IGM. Note that for models UVB3 and UVB4, where the IGM has a large neutral fraction, identifying LLSs becomes ambiguous. At $z=7.085, \Delta z=1$ corresponds to $\sim 43.8 \mathrm{Mpc}$.

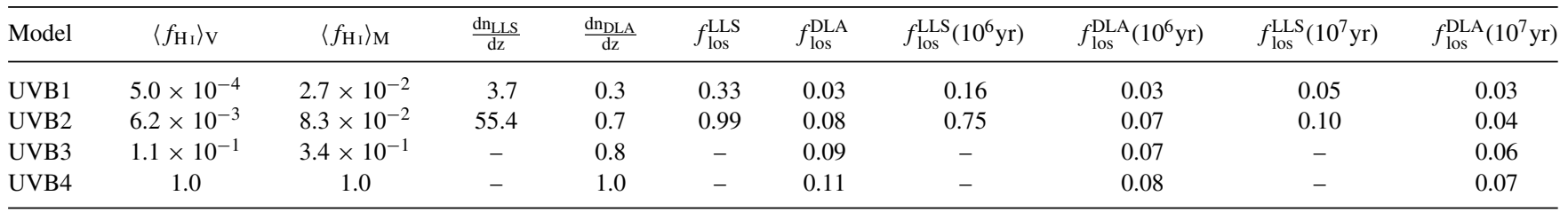

sources (Lidz et al. 2007; Wyithe, Bolton \& Haehnelt 2008). These models are thus designed to explore a range of different initial $\mathrm{HI}$ fractions in the IGM close to the quasar, but are not representative of global reionization histories.

As we only compute the radiative transfer for the quasar ionizing radiation, we also add a simple prescription for regions which are self-shielded from the ionizing background. Assuming that the typical size of an $\mathrm{HI}$ absorber is the Jeans scale (Schaye 2001), the hydrogen number density where an $\mathrm{HI}_{\mathrm{I}}$ absorber begins to self-shield can be approximated as $n_{\mathrm{H}} \simeq 3.6 \times$ $10^{-3} \mathrm{~cm}^{-3}\left(\Gamma_{\mathrm{H}_{\mathrm{I}}} / 10^{-12} \mathrm{~s}^{-1}\right)^{2 / 3}\left(T / 10^{4} \mathrm{~K}\right)^{2 / 15}$. We use this expression to compute the density threshold at which hydrogen is self-shielded prior to being ionized by the quasar, and set the background photoionization rate to zero in these regions. The weak temperature dependence is computed self-consistently using the gas temperature in our simulations. The lower panel of Fig. 2 displays the resulting neutral hydrogen fraction averaged over all 100 sightlines assuming $t_{\mathrm{q}}=10^{7} \mathrm{yr}$ and including (left-hand panel) and excluding (right-hand panel) ionizing radiation from the quasar. The grey curve in the right-hand panel also shows the $\mathrm{H}_{\mathrm{I}}$ fraction for UVB1 excluding the self-shielding model. Self-shielded regions become rapidly more common for ultraviolet background (UVB) models with decreasing $\mathrm{H}$ I photoionization rates, increasing the volume averaged neutral fraction and producing a patchy ionization structure along each sightline. A summary of the average $\mathrm{H}_{\mathrm{I}}$ fractions and incidence of optically thick absorbers in all models which include the self-shielding prescription is given in Table 1. Note that the quasar photoionizes optically thick absorbers in its vicinity, significantly reducing the incidence of these systems. However, the impact of this process is reduced for shorter quasar ages or the smaller ionizing photon mean free path associated with a larger IGM neutral fraction.

\section{RESULTS}

We compare the measured transmission profile around ULAS $\mathrm{J} 1120+0641$, described in detail by M11, to two of our simulated sightlines in Fig. 3. For each sightline we consider quasar ages of $10^{6}$ and $10^{7} \mathrm{yr}$, and for each quasar age the effect of optically thick absorbers is illustrated by models which include and exclude the self-shielding approximation. This qualitative comparison highlights an important point: for an initially neutral IGM (UVB4,
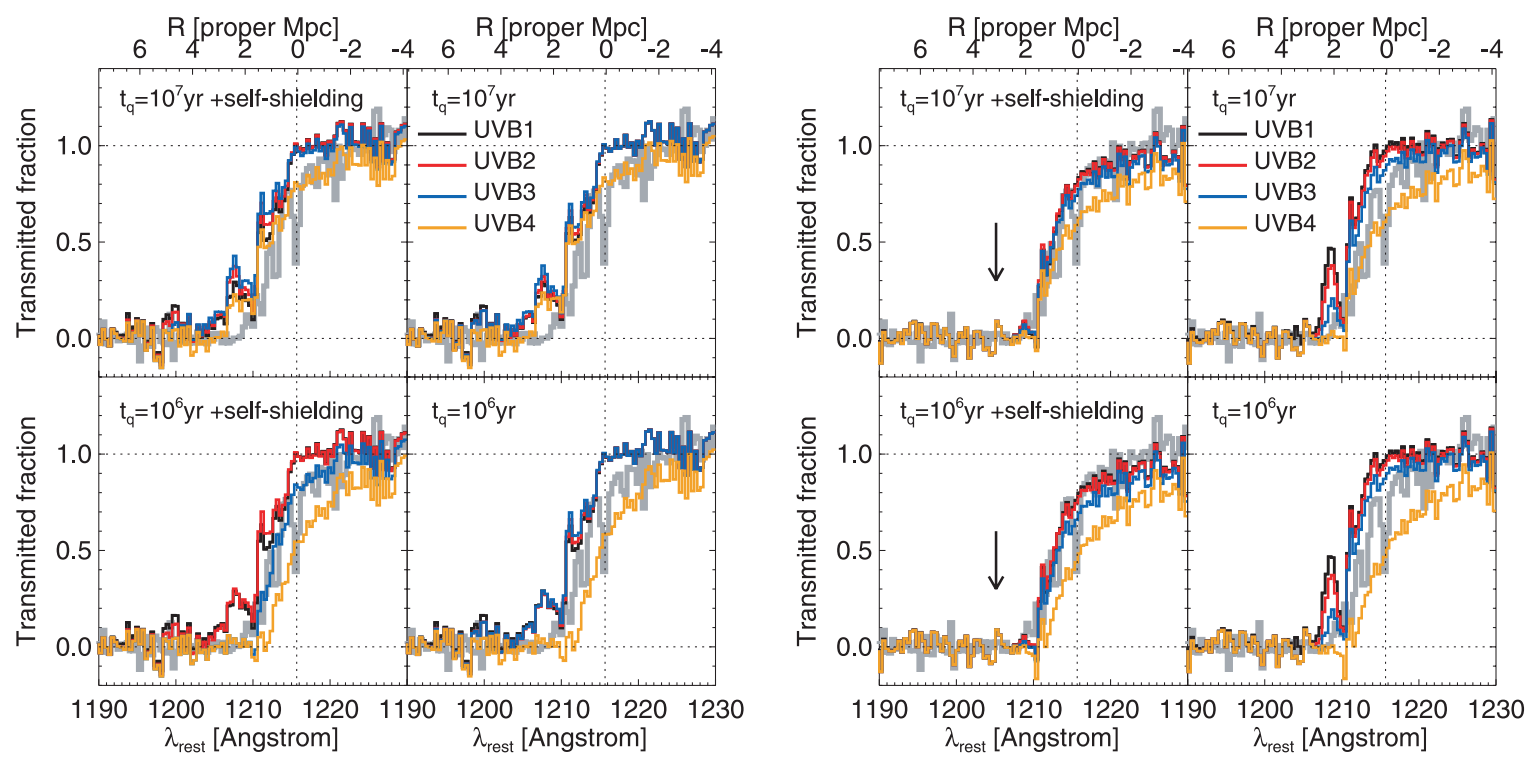

Figure 3. Simulated quasar spectra constructed using the four different UVB models displayed in Fig. 2. The spectra are processed to mimic the M11 spectrum by convolving with a Gaussian instrument profile with FWHM $=240 \mathrm{~km} \mathrm{~s}^{-1}$, rebinning on to pixels of width $0.4 \AA$ (rest frame) and adding Gaussian distributed noise using the observed noise array. The observed transmission of ULAS J1120+0641 is shown by the light-grey curve in each panel. Left: sightline 1. Note the impact of the red GP damping wing on the spectrum for models with a fully neutral IGM (orange curves). Right: sightline 2 . In this sightline a prominent red damping wing is seen for all UVB models when the self-shielding prescription is included. A DLA with $N_{\mathrm{H}} \simeq 10^{20.5} \mathrm{~cm}^{-2}$ is present at the position marked by the arrow. 

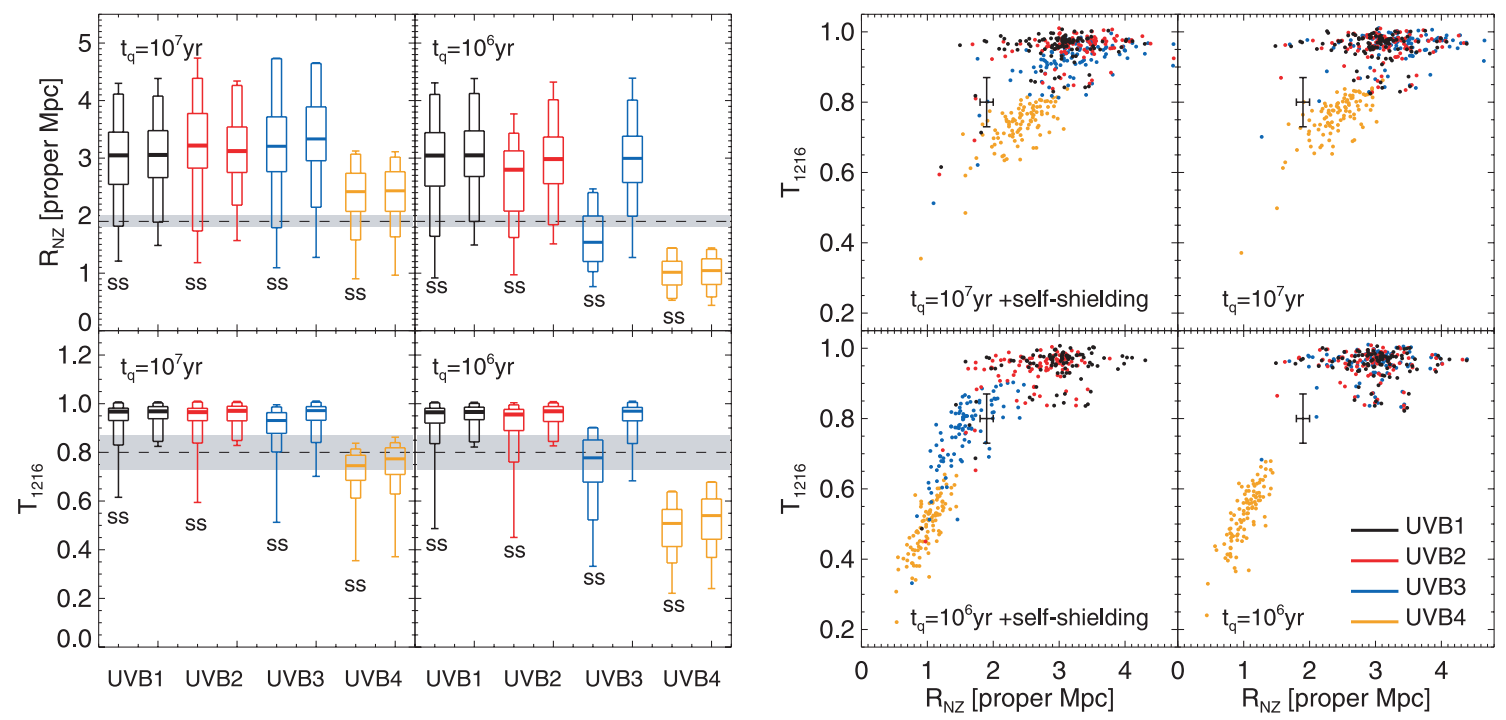

Figure 4. Left: box-whisker plots of the Ly $\alpha$ near zone size (upper panels) and transmitted fraction at the Ly $\alpha$ rest-frame wavelength (lower panels) measured from synthetic quasar spectra. For each UVB model the distributions are shown with (SS) and without the self-shielding prescription. The thick horizontal line in each box displays the median of the distribution, and the vertical extent of the broad and narrow boxes correspond to 68 and 95 per cent of the distribution around the median, respectively. The whiskers display the range of the simulated data. The horizontal dashed line and grey shaded region in each panel displays the measurement for ULAS J1120+0641. Right: scatter plots of the transmission at the Ly $\alpha$ rest-frame wavelength, $T_{1216}$, against near zone size, $R_{\mathrm{NZ}}$. The data point with error bars corresponds to ULAS J1120+0641.

orange curves) or a partially neutral IGM (UVB3, blue curves) and a short quasar bright phase $\left(10^{6} \mathrm{yr}\right)$ a prominent damping wing with absorption extending redward of rest-frame Ly $\alpha$ is present. However, optically thick absorbers will also have an impact on the near zone transmission profile redward of Ly $\alpha$ if a collapsed neutral region is close to the quasar; the presence of a damped Ly $\alpha$ absorber (DLA) with $N_{\mathrm{H}_{\mathrm{I}}} \simeq 10^{20.5} \mathrm{~cm}^{-2}$ at $3.1 \mathrm{Mpc}$, marked by the arrow in the right main panel, demonstrates this.

We may consider the relative likelihood of these possibilities by analysing the transmission profiles for all 100 of our simulated spectra. Considering the moderate signal-to-noise ratio and low resolution of the observed spectrum of ULAS J1120+0641 and the difficulty of continuum fitting due to the complexity of the intrinsic Ly $\alpha$ and $\mathrm{N} v$ emission, we use two simple properties to characterize the transmission profile for a quantitative comparison. These are: the distance from the quasar where the transmitted fraction first falls below 10 per cent, $R_{\mathrm{NZ}}$ (Fan et al. 2006), and the transmitted fraction at the rest-frame Ly $\alpha$ wavelength, $T_{1216}$. These are measured after smoothing the spectra with a box car window of width $2.4 \AA$ (rest frame) to remove fluctuations on smaller scales due to noise and absorption lines. The corresponding values ${ }^{2}$ for ULAS J1120+0641 are $R_{\mathrm{NZ}}=1.9 \pm 0.1 \mathrm{Mpc}$ and $T_{1216}=0.80 \pm 0.07$ after removing the intrinsic absorption line at rest frame $\operatorname{Ly} \alpha$.

Fig. 4 displays box-whisker and scatter plots of the distribution of these two quantities. Both quantities not only depend on the assumed UVB model but also on how long the quasar has been emitting ionizing photons. For example, if self-shielding is included and the quasar has been bright for only $10^{6} \mathrm{yr}$, both quantities for UVB3 are strongly affected by neutral gas in Lyman limit systems (LLSs). Note also the extended tail in the distribution of $R_{\mathrm{NZ}}$ and $T_{1216}$ when self-shielding is included for UVB1 and UVB2. This arises due to the stochastic occurrence of LLSs and DLAs close to the quasar, and

\footnotetext{
${ }^{2}$ The measurements include a systematic error due to the uncertain continuum placement (see fig.4 in M11).
}

is more prominent the shorter the time the quasar has been bright and thus able to photoionize proximate optically thick absorbers. Fig. 4 suggests that the near zone around ULAS J1120+0641 is due to either (i) a partially (fully) neutral surrounding IGM with $\left\langle f_{\mathrm{H}_{\mathrm{I}}}\right\rangle_{\mathrm{V}} \sim$ 0.1 (1.0) and a quasar bright phase of $\sim 10^{6} \mathrm{yr}\left(10^{7} \mathrm{yr}\right)$ or (ii) the presence of a high column density H I system in close proximity to the quasar. It is important to note, however, that (i) and (ii) are not mutually exclusive. In our modelling an abundance of optically thick absorbers close to a quasar is a generic prediction due to the shorter ionizing photon mean free path expected when the IGM is only partially ionized. Our simulations confirm the suggestion of M11 that the spectrum of ULAS J1120+0641 is consistent with an already very highly ionized IGM $\left(\left\langle f_{\mathrm{H}_{\mathrm{I}}}\right\rangle_{\mathrm{V}} \sim 10^{-3}-10^{-4}\right)$ if a DLA lies close to the quasar. However, this occurs in only $\sim 5$ per cent of our sightlines for $t_{\mathrm{q}}=10^{6}-10^{7} \mathrm{yr}$. A fully neutral IGM for $t_{\mathrm{q}}=10^{6} \mathrm{yr}$ is ruled out by the near zone size, but is consistent with the observed transmission for a bright phase duration of $10^{7} \mathrm{yr}$. However, ionization by lower luminosity sources expected to cluster around a massive quasar host halo (Lidz et al. 2007; Wyithe et al. 2008) may make this extreme possibility less likely in practice.

We should caution, however, that if we have underestimated the number of proximate DLAs present at $z \simeq 7$, if the ionizing spectrum of the quasar is much softer than assumed here, or if the environment of the quasar host halo is significantly overdense out to $2 \mathrm{Mpc}$ from the quasar (but see Calverley et al. 2011), then we will overestimate the likelihood of a significantly neutral surrounding IGM and/or underestimate the age of the quasar.

\section{DISCUSSION AND CONCLUSIONS}

We have presented a large suite of realistic synthetic transmission spectra which model a variety of evolutionary histories for the ionization state of the IGM surrounding the quasar ULAS J1120+0641. The transmission profiles depend on the age of the quasar, the photoionization rate in the surrounding IGM and the presence of high column density absorbers arising from collapsed, high-density 
regions. For the same initial conditions for the IGM ionization state the $\operatorname{Ly} \alpha$ transmission profile varies greatly due to the stochasticity of the optically thick absorbers and IGM density distribution along different sightlines.

Inferences regarding the ionization state of the surrounding IGM for Ly $\alpha$ transmission around ULAS J1120+0641 are correlated with the duration of the optically bright phase of the quasar. For a short bright phase duration of $10^{6} \mathrm{yr}$, the data are consistent either with an IGM with a volume averaged neutral fraction $\left\langle f_{\mathrm{H}_{\mathrm{I}}}\right\rangle_{\mathrm{V}} \sim 0.1$ or a highly ionized IGM with $\left\langle f_{\mathrm{H}_{\mathrm{I}}}\right\rangle_{\mathrm{V}} \sim 10^{-3}$ to $10^{-4}$ and a proximate DLA. On the other hand, a bright phase of $10^{7} \mathrm{yr}$ is consistent with both a highly ionized IGM and proximate DLA or a fully neutral IGM. However, we find only $\sim 5$ per cent of our simulated sightlines exhibit a proximate DLA capable of producing a red damping wing, implying the former scenario is less likely (but see also the caveats in Section 4). In either case, this suggests the quasar has not been bright for significantly more than $\sim 10^{7} \mathrm{yr}$; a longer bright phase will produce a near-zone which is too large, either by enlarging the quasar $\mathrm{H}$ II region or photoionizing progressively more proximate optically thick absorbers. A time-scale of $10^{7} \mathrm{yr}$ is still a factor of 5 less than the e-folding time of a black hole growing at the Eddington rate and radiating with 10 per cent efficiency, as might be expected for a moderately spinning black hole. For a rapidly spinning black hole the discrepancy would be even larger (Sijacki, Springel \& Haehnelt 2009). Unless the black hole grows in an earlier optically obscured phase, this adds to the challenge of building up a black hole with an estimated mass of $2 \times 10^{9} \mathrm{M}_{\odot}$ so early in the history of the Universe. A higher quality spectrum will hopefully exclude or confirm the possible presence of a proximate DLA by enabling the identification of associated metal lines and aid in narrowing constraints on the neutral fraction of the surrounding IGM.

More general conclusions regarding the ionization state of the IGM at $z \sim 7$ are speculative due to the large fluctuations in the ionization state expected on scales much larger than probed by the near zone of ULAS J1120+0641 (Mesinger \& Furlanetto 2008). If the neutral fraction in the general IGM at $z \sim 7$ is indeed $\left\langle f_{\mathrm{H}_{\mathrm{I}}}\right\rangle_{\mathrm{V}} \sim 0.1$ this is nevertheless consistent with a constant or weakly decreasing ionizing emissivity between $z \sim 6$ and 7 . The rapid change in the photoionization rate by two orders of magnitude or more in our models is balanced by a similar change in the number of LLSs and thus the mean free path of ionizing photons (e.g. McQuinn, Oh \& Faucher-Giguere 2011). Since a constant or perhaps even rising emissivity is required for the completion of reionization at $z \gtrsim 6$ (Miralda-Escudé 2003; Bolton \& Haehnelt 2007b), this may be compatible with the possibility that reionization was yet to fully complete by $z=6$ (Mesinger 2010). Lastly, the possibility of a significant neutral fraction in the IGM surrounding ULAS J1120+0641 make it an excellent target for searching for extended Ly $\alpha$ emission from the expanding ionization front (Cantalupo, Porciani \& Lilly 2008) as well as studies of the IGM ionization state using the redshifted $21-\mathrm{cm}$ transition.

\section{ACKNOWLEDGMENTS}

JSB acknowledges the support of an ARC Australian post-doctoral fellowship (DP0984947) and thanks Stuart Wyithe for valuable comments.

\section{REFERENCES}

Alvarez M. A., Abel T., 2007, MNRAS, 380, L30

Becker G. D., Rauch M., Sargent W. L. W., 2007, ApJ, 662, 72

Bolton J. S., Haehnelt M. G., 2007a, MNRAS, 374, 493

Bolton J. S., Haehnelt M. G., 2007b, MNRAS, 382, 325

Bolton J. S. et al., 2010, MNRAS, 771

Calverley A. P., Becker G. D., Haehnelt M. G., Bolton J. S., 2011, 412, 2543

Cantalupo S., Porciani C., Lilly S. J., 2008, ApJ, 672, 48

Carilli C. L. et al., 2010, ApJ, 714, 834

Fan X. et al., 2006, AJ, 131, 1203

Gunn J. E., Peterson B. A., 1965, ApJ, 142, 1633

Lawrence A. et al., 2007, MNRAS, 379, 1599

Lidz A. et al., 2007, ApJ, 670, 39

McQuinn M., Oh S. P., Faucher-Giguere C., 2011, ApJ, preprint (arXiv:1101.1964)

Maselli A., Gallerani S., Ferrara A., Choudhury T. R., 2007, MNRAS, 376, L34

Maselli A., Ferrara A., Gallerani S., 2009, MNRAS, 395, 1925

Mesinger A., 2010, MNRAS, 407, 1328

Mesinger A., Furlanetto S. R., 2008, MNRAS, 385, 1348

Mesinger A., Haiman Z., 2004, ApJ, 611, L69

Miralda Escudé J., 2003, ApJ, 597, 66

Miralda Escude J., Rees M. J., 1998, ApJ, 497, 21

Mortlock D. J. et al., 2011, Nat, 474, 616

Schaye J., 2001, ApJ, 559, 507

Sijacki D., Springel V., Haehnelt M. G., 2009, MNRAS, 400, 100

Springel V., 2005, MNRAS, 364, 1105

Willott C. J. et al., 2010, AJ, 140, 546

Wyithe J. S. B., Bolton J. S., 2011, MNRAS, 412, 1926

Wyithe J. S. B., Loeb A., 2004, Nat, 432, 194

Wyithe J. S. B., Bolton J. S., Haehnelt M. G., 2008, MNRAS, 383, 691

This paper has been typeset from a $\mathrm{T}_{\mathrm{E}} \mathrm{X} / \mathrm{L} \mathrm{T}_{\mathrm{E}} \mathrm{X}$ file prepared by the author. 